\title{
Visual acuity in low birth weight (1500-2500 g) neonates
}

\author{
A.J.M. Hermans ${ }^{a}$, J. Van Hof-van Duin ${ }^{a}$ and \\ A.M. Oudesluys-Murphy \\ ${ }^{a}$ Department of Physiology I, Erasmus University Rotterdam, PO Box 1738, 3000 DR Rotterdam, and \\ ${ }^{b}$ Department of Paediatrics, Zuiderziekenhuis, Rotterdam, (The Netherlands)
}

(Received 15 August 1991; revision received 20 January 1992; accepted 21 January 1991)

\section{Summary}

Binocular grating acuity was tested in 138 low birth weight (LBW) neonates (birth weights ranging from 1500 to $2500 \mathrm{~g}$ ) by means of the prototype version of the Acuity Card Procedure. No surrounding screen was used. Mean visual acuity of 107 neonates successfully assessed at mean corrected ages of -1.9 weeks $( \pm 1.9$ weeks) amounted to 0.58 cycles/degree (S.D. 0.71 octaves). Success rate was $77.5 \%$. Mean postnatal age was 2.3 weeks ( \pm 1.6 weeks). Acuity values of various subgroups ranged between 0.68 cycles/degree (S.D. 1.3 octaves) in low-risk, small for gestational age (SGA) preterms $(n=7)$, to 0.56 cycles/degree (S.D. 0.7 octaves) in SGA fullterms $(n=34)$, independent whether at low-or at high-risk. These differences were not significant, although with multiple regression analysis with adjustment for corrected age of testing, mean acuity of low-risk preterms was slightly better than of low-risk fullterms $(P=0.055)$. No significant change of acuity over corrected age could be demonstrated, except for a slight progress $(r=0.57 ; P<0.05)$ in the subgroup of 13 low-risk fullterms. The high variability of acuity values in neonates and the slow acuity development at term age hamper assessment of differences between various subgroups of neonates.

Key words: visual acuity; development; neonates; preterms; fullterms; SGA; AGA; low birth weight; low-risk

Correspondence to: J. Van Hof-van Duin, Department of Physiology I, Erasmus University Rottcrdam, PO Box 1738, 3000 DR Rotterdam, The Netherlands.

0378-3782/92/\$05.00 C 1992 Elsevier Scientific Publishers Ireland Ltd.

Printed and Published in Ireland 


\section{Introduction}

There is no good agreement across laboratories and techniques on neonatal visual acuity values. Binocular visual acuity estimates of 1 cycle/degree have been described in healthy fullterm newborns using the forced-choice preferential looking (FPL) technique $[25,27]$, the Acuity-Card procedure $[8,9]$ as well as with pattern visual evoked potential (VEP) techniques [3,4,23]. One study by Atkinson and Braddick [2] claimed to find no significant differences between VEP and FPL acuity estimates using carefully matched stimulus parameters from repeated measures of a single infant from birth to 3 months of age. On the other hand, Brown and Yamamoto [7] were unable to assess visual acuity in neonates with Teller acuity cards, but reported values of 0.69 cycles/degree using modified, so called 1 -aperture acuity-cards. Furthermore, Birch et al. [6] reported a VEP acuity of 3.1 cycles/degree at 36 weeks post conceptional age, whereas Norcia and Tyler [20] described VEP-acuity values of 4.5 cycles/degree at 40 weeks post-conceptional age. At present no behavioural visual acuity norms obtained with 2 -aperture acuity cards are available for (healthy) preterm infants in the neonatal period.

There is no general agreement on the rate of improvement of acuity over the first two months of life. While some studies $[3,23]$ showed little or no improvement during the first $1-2$ postnatal months, other authors reported a smooth transition in visual development from the preterm through the neonatal and early postnatal period with improvement of visual acuity of $0.25-0.45$ octaves per month $[6,7,20]$.

Some beneficial effects of extra-visual experience in low-risk preterms have been described during the first year of life. The effects were however limited and more pronounced in the more preterm (gestation < 31 weeks) infants $[21,25,26]$. As yet no advantageous effect of extra-visual experience in preterms has been demonstrated in the period round term age.

Preterm infants and infants who suffered perinatal insults are at risk of developing impaired visual acuity. Although visual acuity deficits could be demonstrated as early as 6 weeks corrected age $[10,13,28]$, visual acuity differences between healthy and hospitalised newborns without neurological and ophthalmological abnormalities appeared not to be significant at term [7].

There is evidence from the literature that intra-uterine growth retardation (IUGR) adversely effects fetal brain development [5], although compensation mechanisms may exist $[1,24]$. Long term effects of IUGR on the visual system have not been demonstrated [28], short term effects remain to be elucidated.

Earlier studies in our laboratory have described visual deficits in very low birth weight (VLBW, $<1500 \mathrm{~g}$ ) infants during the first years of life [28]. Visual development in infants with birth weights ranging from 1500 to $2500 \mathrm{~g}$ is as yet unknown, although this birth weight category is considerably larger than that of VLBW infants. As part of a prospective study on visual development during the first 2 years of life we assessed binocular visual acuity in newborns with birth weights ranging from $1500-2500 \mathrm{~g}$. The aims of this study were to establish visual acuity norms in neonates, to investigate whether or not a progress in acuity development can be demonstrated in the neonatal period and to examine the possibilities of assessing differences in visual acuity between at-risk groups during the neonatal period. Visual 
acuity was tested using the acuity-card procedure, a procedure successfully used for testing acuity in fullterm and preterm infants as well as in pediatric patients $[9,17,19]$.

\section{Subjects}

Subjects $(n=107)$ were part of the study population $(n=258)$ consisting of infants, born between January 15, 1987 and October 22, 1989. All infants were admitted to the pediatric department of the Zuiderziekenhuis at Rotterdam immediately after delivery or during the neonatal period. The Zuiderziekenhuis has a maternity clinic and a high care neonatal care unit (level 2 hospital 22, see Ref. 22) and provides medical care for part of the city of Rotterdam and its immediate surroundings. The criteria for entry into the study were birth weight (from $1500 \mathrm{~g}$ up to and including $2500 \mathrm{~g}$ ) and informed consent from the parents. The study was approved by the Medical Ethical Committee of the Zuiderziekenhuis and the Erasmus University Rotterdam. During the neonatal period binocular visual acuity testing was limited to 138 infants, due to the medical condition and/or sleepiness of the neonates, and because of time constraints as a result of feeding procedures; in 5 cases parents refused to participate in the study. Eighty-eight infants were inborn; transport to a neonatal intensive care unit was necessary in 15 infants. Mean duration of hospitalization was 3.98 weeks ( \pm 3.5 weeks).

Medical data were obtained from the neonates pediatric records which included the mothers' obstetrical information. For preterms and fullterms, duration of gestation was calculated from mother's last menstruation, obstetrical data and, where available, early prenatal ultrasound scans. The gestational duration was uncertain in 5 infants. In 4 of these 5 infants gestational duration was assessed by means of early prenatal ultrasound scans as well as, or in some cases only by means of a clinical assessment of gestational age as described by Dubowitz et al. [11]. Neither of these methods had been used in one infant and the uncertainty in the duration of gestation was 2 weeks.

Infants were divided into 70 preterms (gestation $<37$ completed weeks) and 37 fullterms (gestation from 37 completed weeks to 42 weeks) according to WHOdefinitions [29].

Classification into small-for-gestational age (SGA) and appropriate-forgestational age (AGA) was made according to 'Amsterdam growth charts' using the 10 th percentile as cutoff point. These charts are based on the Dutch population and contain corrections for sex and parity [15]. Figure 1 shows the distribution of the newborns according to birth weight and duration of gestation. Seventy infants were born preterm (53 AGA and 17 SGA) and 37 fullterm ( 3 AGA and 34 SGA).

Division into optimally healthy (low-risk) and infants at risk (high-risk) was made according to criteria used in earlier studies [25]. In order to establish normative values for visual acuity it was necessary to exclude all infants who would possibly have been exposed to perinatal or neonatal stress conditions. The criteria for highrisk used in this study were the need for obstetrical intervention because of fetal distress, an Apgar score of 6 or less at $5 \mathrm{~min}$, mechanical ventilation for more than one day, oxygen treatment for more than 7 days, bilirubin concentration in serum 


\section{DISTRIBUTION GESTATION}
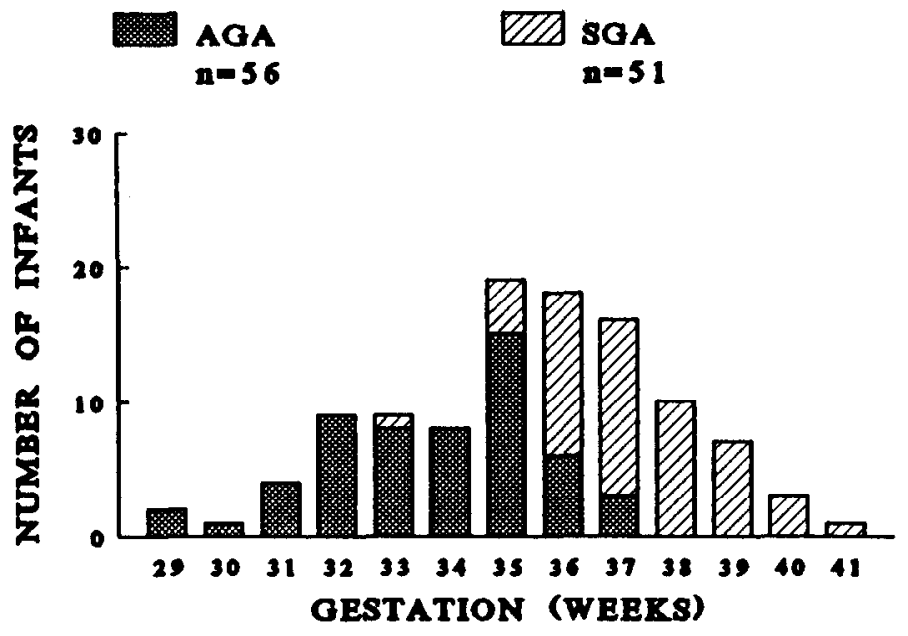

\section{DISTRIBUTION BIRTH WEIGHT}
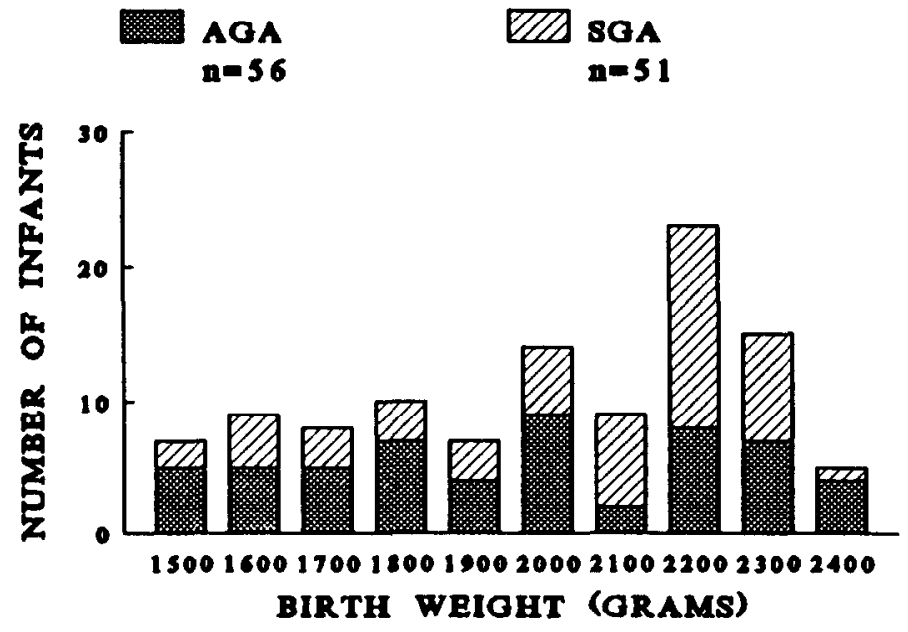

Fig. 1. Distribution of $107 \mathrm{LBW}$ neonates according to gestation and birth weight.

$\geq 250 \mathrm{mmol} / \mathrm{l}$, bradycardia needing medical treatment and clear neurological symptoms in the neonatal period. Neonates were classified as neurologically normal $(n=98)$ in the absence of clear neurological symptoms in the neonatal period such as jitteriness, floppiness, hypo- or hypertonia, hyperexcitability and convulsions, and as abnormal $(n=9)$ in the presence of such symptoms. None of the infants showed abnormalities in eye position or eye movements. The criterium 'obstetrical intervention because of fetal distress' included conditions such as caesarian section because 
TABLE I

Division of study population $(n=107)$ into subgroups.

\begin{tabular}{lcc}
\hline 70 Preterms & 53 AGA & 17 SGA \\
\hline 33 Low-risk & 26 & 7 \\
37 High-risk & 27 & 10 \\
37 Fullterms & 3 AGA & 34 SGA \\
\hline 13 Low-risk & 1 & 12 \\
24 High-risk & 2 & 22 \\
\hline
\end{tabular}

of (pre)eclampsia and solutio placentae, induction of labour because of growth retardation, and termination of delivery by the use of forceps or vacuum extraction.

As a standard procedure all infants who required oxygen treatment for more than $4 \mathrm{~h}$, were seen by an ophthalmologist during the neonatal period. In none of the examined infants signs of retinopathy of prematurity were present. Infants with persistent bradycardia or clear abnormal neurological signs underwent brain CT-scan examination. No gross abnormalities were seen. The subject group consisted of 46 low-risk and 61 high-risk infants. (Table I). Table II gives an overview of general characteristics and selected risk factors of the 107 reliably tested infants.

The infants were either tested in the nursery or in the high care neonatal unit. Ninety-one infants were tested in the nursery out of their bed and 5 infants in the high care neonatal unit out of bed or incubator. Two infants were tested in the nursery in bed and 9 infants in the high care neonatal unit in bed or incubator.

\section{Methods}

Visual acuity was tested with the prototype Acuity Card Procedure [16] using the version described by Mohn and van Hof-Van Duin [17]. The procedure was adjusted slightly to make it more suitable for testing neonates. The method is based on the preference babies show for a pattern when presented in a neutral surrounding [12]. Two-aperture stimulus cards were used. The procedure was carried out without a surrounding screen. Details of the procedure have been described earlier [19].

At a test distance of $30-40 \mathrm{~cm}$ the baby is shown a series of gray cards which contain two circular targets. One of these targets was the test stimulus, the other consisted of fine stripes unresolvable to the infant. Test stimuli were high contrast black and white gratings of equal space averaged luminance $\left(14 \mathrm{~cd} / \mathrm{m}^{2}\right)$ with stripe widths decreasing in half-octave steps (an octave is a doubling or halving of stripe width). Stripe widths of the gratings ranged from 0.19 cycles/degree to 48 cycles/ degree. The test set contained 16 cards, with an additional card with two blank targets to gain an impression of the infant's reaction to an unresolvable grating. Visual acuity was defined as the smallest stripe width which elicited a staring preference in the baby as judged by an experienced observer, and was expressed in cycles/degree or minutes of arc. 
TABLE II

General characteristics and *factors used in multiple regression analysis in $n=107$ LBW neonates.

\begin{tabular}{ll}
\hline Gender* & $\begin{array}{l}\text { Male, } n=61 \\
\text { Female, } n=\end{array}$ \\
Race* & $\begin{array}{l}\text { Caucasian, } n \\
\text { Non-caucasia }\end{array}$ \\
Birth weight (g)* & $2047 \pm 259$ \\
Gestation (weeks)* & $35.78 \pm 2.43$ \\
Preterms* & $n=70$ \\
Fullterms & $n=37$ \\
AGA* & $n=56$ \\
SGA & $n=51$
\end{tabular}

Duration of hospital stay

Mean 3.98 weeks

S.D. 3.50 weeks

Place of birth

Inborn, $n=88$

Outborn, $n=19$

Neonatal transport to neonatal intensive care unit

Yes, $n=15$

No, $n=92$

Yes, $n=22$

Multiple pregnancy*

No, $n=85$

Mode of delivery*

Vaginal occip., $n=81$

Vaginal breech, $n=11$

Caesarian section, $n=15$

Maternal hypertensive disorders of pregnancy*

Low/high risk*

Obstetrical intervention because of

Fetal distress

Apgar score at $5 \mathrm{~min}$

Neonatal neurological

examination

Mechanical ventilation

Oxygen treatment

Yes, $n=28$

No, $n=79$

Low risk, $n=46$

High risk, $n=61$

No, $n=70$

Yes, $n=37$

$\leq 6, n=5$

$>6, n=102$

Normal, $n=98$

Abnormal, $n=9$

$\leq 1$ day, $n=103$

$>12$ day, $n=4$

$\leq 7$ days, $n=98$

$>7$ days, $n=9$

Bilirubin

$\leq 250 \mathrm{mmol} / 1, n=97$

$>250 \mathrm{mmol} / \mathrm{l}, n=10$

Bradycardia needing medical treatment

No, $n=87$

Yes, $n=20$

Mortality

$n=3$, one during initial hospital stay 
The babies were tested only when they were awake spontaneously and when their medical condition allowed it. If possible they were taken out of their incubator or bed and held in an upright position with the head supported and the acuity cards presented in front of the baby. Otherwise, they were tested in their incubator or bed lying supine or on one side and judgment of staring preference was made while the observer was standing beside the incubator or bed. In the supine position the test cards were held above the babies. When the baby was lying on one side test stimulus and 'blank' could not be presented simultaneously, but were held in front of the baby's face alternately. Reactions of the baby were observed through a small peephole in the centre of each card, or, when the baby was lying in the bed or incubator, by looking over the card directly at the baby's face.

It usually took several seconds before a staring behaviour was clicited by a grating. The infant would slowly turn his or her eyes and or head towards the test stimulus and kept staring in that direction. Reactions to larger stripe widths could be difficult to judge because the test stimulus did not contain enough stripes. In such cases the test stimulus containing six stripes $(0.26$ cycles/degree) was presented. When reactions could be judged confidently, cards were presented in a staircase procedure, in which stripe widths decreased in half octaves. Each card was rotated by the observer to position the stimulus grating on the opposite side, whereafter a new judgment as to the location of the grating was made. Before presentation of the grating the observer was always blind to the location of the grating on the card. When the infant was judged not to see a test stimulus, gratings with coarser and finer stripe widths were presented successively (in half-octave steps) until the observer had made at least two judgments of the finest visible grating. Only data from infants who remained fully awake throughout the procedure and in whom a confident judgment could be made were included in the analysis. The whole procedure took about 8-10 min and about 20 trials were usually required.

Statistical analysis included univariate analysis (Student's $t$-test, Chi-square with Yates correction, Pearson's correlation coefficient, Spearman's rank correlation coefficient, sign test), one-way analysis of variance, and a multiple regression analysis procedure. Analysis of residuals did not reveal violations of the assumptions made in the multiple regression procedure nor were signs of interactions between risk factors present. Statistical tests were performed at a significance level of 5\%.

\section{Results}

Binocular visual acuity was assessed in the newborn period in 107 out of 138 infants (success rate $77.5 \%$ ). Corrected ages varied from -6.14 to 3.29 weeks and postnatal ages from 0.29 to 7.71 weeks. Thirty newborns were tested repeatedly, 23 infants twice, 3 infants 3 times, 2 infants 4 times, 1 infant 6 times and 1 infant 7 times.

\section{Mean binocular visual acuity}

Mean binocular visual acuity was 0.58 cycles/degree (S.D. 0.71 octaves). In these analyses only the first confident test result of each of the 107 infants is included. Apart from acuity estimates of 1.24 cycles/degree \pm 0.87 octaves, obtained in 2 highrisk AGA fullterms, acuity values ranged from 0.52 cycles/degree \pm 0.65 octaves in 
low-risk fullterms to 0.68 cycles/degree \pm 1.31 octaves in low-risk SGA-preterms (Table III). Mean corrected age of testing was -1.91 weeks \pm 1.88 and ranged from -6.14 weeks to 3.29 weeks.

No differences in mean visual acuity were observed between preterms and fullterms, between AGA and SGA, or between low-and high-risk infants. In addition, no differences were seen between low-and high risk preterms, fullterms, AGA and SGA-infants. Univariate and multiple regression analysis with adjustment for corrected age did not reveal differences in mean visual acuity between the subgroups AGA-preterms, SGA-preterms, AGA-fullterms and SGA fullterms.

Within the low-risk group a marginally significant $(P=0.055)$ difference could be demonstrated between preterms and fullterms, with low-risk preterms having 0.6 octaves higher mean visual acuities compared to low-risk fullterms (multiple regression analysis with adjustment for corrected age).

Development of visual acuity

The presence or absence of progress in acuity development during the neonatal period was examined in several ways.

Firstly, mean visual acuity using the first confident acuity estimate of each infant $(0.58 \mathrm{cycles} / \mathrm{degree} \pm 0.71)$ was compared to that obtained at a corrected age as close as possible to term age $(0.60$ cycles/degree \pm 0.7 octaves $)$. However, mean corrected

TABLE III

Mean binocular grating acuity and mean postnatal and corrected ages of testing in 107 LBW-neonates and subgroups.

\begin{tabular}{lcllllll}
\hline & $n$ & $\begin{array}{l}\text { Acuity } \\
\text { (cycles/ } \\
\text { degrees) }\end{array}$ & $\begin{array}{l}\text { (S.D.) } \\
\text { (octaves) }\end{array}$ & $\begin{array}{l}\text { Postnatal } \\
\text { age } \\
\text { (weeks) }\end{array}$ & (S.D.) & $\begin{array}{l}\text { Corrected } \\
\text { age } \\
\text { (weeks) }\end{array}$ & (S.D.) \\
\hline Study population & 107 & 0.58 & $(0.71)$ & 2.31 & $(1.63)$ & -1.91 & $(1.88)$ \\
Preterms & 70 & 0.60 & $(0.73)$ & 2.78 & $(1.68)$ & -2.76 & $(1.60)$ \\
AGA-preterms & 54 & 0.58 & $(0.70)$ & 2.87 & $(1.68)$ & -3.17 & $(1.39)$ \\
SGA-preterms & 16 & 0.67 & $(0.84)$ & 2.50 & $(1.60)$ & -1.46 & $(1.52)$ \\
Fullterms & 37 & 0.56 & $(0.68)$ & 1.44 & $(1.11)$ & -0.03 & $(1.22)$ \\
AGA-fulltermas & 3 & 0.55 & $(1.13)$ & 1.48 & $(1.58)$ & -1.10 & $(1.57)$ \\
SGA-fullterm & 34 & 0.56 & $(0.65)$ & 1.43 & $(1.10)$ & -0.23 & $(1.19)$ \\
AGA & 56 & 0.58 & $(0.71)$ & 2.79 & $(1.69)$ & -3.10 & $(1.47)$ \\
SGA & 51 & 0.59 & $(0.73)$ & 1.79 & $(1.1)$ & -0.64 & $(1.42)$ \\
Low-risk & 46 & 0.59 & $(0.78)$ & 1.81 & $(1.32)$ & -2.25 & $(2.02)$ \\
Low-risk PT & 33 & 0.62 & $(0.83)$ & 2.04 & $(1.43)$ & -2.99 & $(1.76)$ \\
Low-risk FT & 13 & 0.52 & $(0.65)$ & 1.21 & $(0.77)$ & -0.36 & $(1.28)$ \\
High-risk & 61 & 0.58 & $(0.66)$ & 2.69 & $(1.74)$ & -1.65 & $(1.75)$ \\
High-risk PT & 37 & 0.58 & $(0.64)$ & 3.43 & $(1.63)$ & -2.55 & $(1.43)$ \\
High-risk FT & 24 & 0.57 & $(0.71)$ & 1.56 & $(1.26)$ & -0.26 & $(1.21)$ \\
\hline
\end{tabular}


age as close as possible to term age was $-1.66 \pm 1.87$ weeks (median -1.57 ), which is only 2 days later than when first test results were used.

Secondly, the first confident test result of each infant was plotted against corrected age and postnatal age of testing respectively using linear regression analysis. Corrected age ranged from -6.14 to 3.29 weeks and postnatal age from 0.29 to 7.14 weeks. A Spearman rank correlation coefficient was calculated instead of Pearson's correlation coefficient in case violations of assumptions were suspected. In low-risk fullterms ( $n=13, r=0.57)$ improvement of visual acuity over corrected age was seen $(P<0.05)$. No signs of progress in acuity development could be demonstrated in the total study population nor in any of the other subgroups.

In a third way of approaching the question whether development of acuity occurred during the neonatal period, results obtaincd in 30 repeatedly tested infants were analysed. The minimum time interval between first and last test session in individual infants was 2 days and the maximum interval 4.71 weeks. A Spearman rank correlation coefficient was calculated in each individual infant to assess development of visual acuity over corrected age. A sign test was applied to decide whether the chance of having more positive values (improvement of visual acuity over corrected age) than negative ones (deterioration of visual acuity over corrected age) in this group of 30 infants was statistically significant. This approach did not reveal relations between repeated measurements over corrected age $(P>0.05)$.

To examine if development of acuity could be demonstrated over a certain period of time, results of infants with test intervals between first and last assessment of at least 2 weeks $(n=9)$, were analysed. In only 3 infants progress in acuity development appeared to be significant ( $P<0.05$, Spearman rank correlation coefficient). No acuity development was seen in infants with test intervals of less than 2 weeks.

Finally, a repeated measurements analysis of variance was performed using data of all the infants, including the results of the repeatedly tested infants. No effects of respectively corrected age and postnatal age on visual acuity were seen $(P>0.05)$.

\section{Relation with perinatal risk factors}

Table II presents an overview of the selected factors. The criteria used for the classification into high-and low-risk infants were also analysed separately to examine their effect on visual acuity. Univariate analysis as well as multiple regression analysis with adjustment for corrected age revealed a (marginally) significant relation $(P=0.067)$ between multiple pregnancy and visual acuity. Infants born from a multiple pregnancy had lower ( 0.32 octaves) mean visual acuities compared with singleton born infants. The factor low/high-risk nor any of the factors used in the risk-classification or the factors gestational duration, birth weight and intra-uterine growth retardation influenced visual acuity in the neonatal period. The factor multiple pregnancy remained (marginally) significant $(P=0.06)$ after adjustment for gestational duration, birth weight, intra-uterine growth retardation and high/low risk in a multiple regression analysis.

Additionally, there was no significant difference in mean visual acuity between in or out of bed or incubator tested infants. However, mean visual acuity of infants tested in the nursery was 0.38 octaves higher than visual acuity of infants tested in the high care neonatal unit (multiple regression analysis with adjustment for cor- 
rected age; $P=0.07)$. The majority of infants tested in the high care unit $(n=14)$ was tested in bed or incubator $(n=9)$. After adjustment for this factor (being tested in bed or incubator) the observed difference in mean visual acuity between nursery and high care unit disappeared.

\section{Discussion}

Mainly due to time constraints as a consequence of feeding procedures in the nursery, it proved impossible to test visual acuity in the total study population $(n=258)$. This may have caused bias. However, the infants in which a confident estimate of visual acuity could be made $(n=107)$ did not differ significantly from the not-tested infants $(n=151)$ except for mean birth weight $(P=0.005)$. Mean birth weight of the reliably tested infants was lower $(2046 \pm 259 \mathrm{~g}$ versus $2137 \pm 246 \mathrm{~g}$ ). None of the other general characteristics and (perinatal) risk factors (Table II), including mean duration of hospitalisation were significantly different. The tested infants can therefore be considered to represent the total study population.

Despite the fact that not all the infant's acuity could have been assessed the method proved to be satisfactory once it was started. A confident estimate of visual acuity could be assessed in 107 out of 138 infants. This high success rate of $77.5 \%$ is in contrast with Brown and Yamamoto's results [7] who did not succeed in testing visual acuity using two-aperture cards, but agrees with reports by Dobson et al. [9]. The method did not take much time (about 8-10 min) and did not disturb nursing procedures. The infants tested in bed or incubator showed similar acuity values as those who could be taken out of bed or incubator; in addition, acuity values between infants tested in the nursery and the high care unit were comparable. The medical conditions which did not allow infants to be taken out of their beds or incubators obviously did not influence acuity. The Acuity Card Procedure can therefore easily be performed as a routine screening method of visual acuity, even in newborns in a nursery.

Acuity values obtained in newborns with birth weights ranging from 1500 to $2500 \mathrm{~g}$ and corrected ages between -6.14 and 3.29 weeks are comparable with those described by Brown and Yamamoto [7], but are significantly lower than reported both in behavioural $[9,19,25,27]$ and in electrophysiological $[6,21]$ studies. Discrepancies in acuity values obtained with electrophysiological and behavioural methods have been reported and discussed earlier (for an overview, including a discussion on the neural basis of infant spatial vision, see [18]). The discrepancies between the present results and the other behavioural studies might possibly be explained by the fact that the medical condition of the LBW neonates did usually not allow to hold them in an upright position and keep them as alert and active as the healthy fullterm neonates reported in the other behavioural studies $[9,19,25,27]$.

Similar to the investigations by Brown and Yamamoto [7] we were unable to demonstrate significant differences in mean visual acuity between high- and low-risk infants. This might be due to the very strict selection criteria used for the classification of the infants. As a consequence our so-called high-risk infant group is likely to contain a certain percentage of infants not particularly at high risk but probably 
more at some minor risk. Subsequently, mean visual acuity of truly high-risk infants could therefore be lower. Be that as it may, our present results do not allow to differentiate the high-risk infants in relation to the acuity estimates and/or variability (Table III).

A (marginally) significant difference $(P=0.055)$ in mcan visual acuity indicating a slight beneficial effect of extra visual experience, could only be demonstrated between low-risk preterm and low-risk fullterm infants. After adjustment for corrected age low risk preterms have 0.6 octaves higher mean visual acuities. Although the number of low-risk fullternıs is rather small $(n=13)$ this finding presents some evidence of an additional beneficial effect of extra visual experience in low-risk preterms in the neonatal period. Such an effect has previously been described during the first year of life by Norcia et al. [21], whereas in the studies by Van Hof-van Duin and Mohn [25,26] some indications but no significant evidence was reported.

Considering norm values for visual acuity in the neonatal period, discrimination between low-risk preterm and low-risk fullterm infants is apparently necessary. Since no differences between any of the other subgroups could be demonstrated, in particular not between AGA and SGA-infants and between low- and high-risk infants, mean visual acuity of the total subject group could be used as norm value for these subgroups. However, one has to bear in mind that truly high-risk infants actually could have lower mean visual acuities.

No significant differences in mean visual acuity were seen when using the first reliable acuity estimates or the test results obtained as close to term age as possible. This may however have been caused by the small difference in corrected age of only 2 days.

Results of repeated measurements of visual acuity in all 30 infants did not show a clear improvement with corrected age. Analysis of data of 9 infants tested with an interval of at least 2 weeks showed some improvement of acuity with age. However, in only 3 infants this improvement was significant. This small number of infants as well as a possible learning effect does not allow firm conclusions about an increase in acuity in this period. On the other hand, the observed similar acuity estimates obtained in infants tested with intervals of less than 2 weeks may be used to underline the intertest reliability of the acuity card procedure in neonates.

Signs of improvement of visual acuity over corrected age round term age could only be demonstrated in low-risk fullterms, particularly in low-risk SGA-fullterms ( $r=0.57$ and 0.52 , respectively). This finding seems to confirm those of Norcia and Tyler [20], Brown and Yamamoto [7] and Birch et al. [6]. However, no development of visual acuity over corrected age was seen in the total study group nor in any of the other subgroups, which agrees with Atkinson et al. [3] and with Porciatti et al. [23]. Since improvement of acuity in the present study was observed in a rather small subgroup $(n=13)$, and a repeated measurements analysis of variance of the total subject group did not reveal signs of improvement of acuity, strong conclusions about development of acuity in the total subject group cannot be drawn.

Considering possible risk factors, birth weight as such has no specific relation with visual acuity in neonates whose birth weights range between 1500 and $2500 \mathrm{~g}$. Apart from a beneficial effect of gestational duration in low-risk preterms compared with low-risk fullterms no other effects of gestation were seen. Confirming results describ- 
ed by Norcia et al. [21], multiple pregnancy was found to be a (marginal) risk factor for lower mean visual acuity, even after correction for gestation, birth weight, IUGR, or being at low- or at high-risk. The nature of multiple pregnancy as a risk factor is still unclear. None of the other analysed risk factors could be identified as to affect visual acuity in the neonatal period.

No pronounced beneficial nor adverse effects of intra-uterine growth retardation (IUGR) on mean visual acuity could be demonstrated. The presumably negative influence of IUGR on fetal brain development [5] is therefore possibly well compensated for by a redistribution of fetal bloodflow in favor of the fetal brain [1,24].

Mean variability of acuity values during the neonatal period amounted to 0.71 octaves. This S.D. is similar to that reported earlier $[8,9,19,25]$ in the neonatal period, but is high compared to variability values of $0.27-0.5$ octaves described in children during the first year of life [19], and to the S.D. of 0.4 octaves in children between 1 and 4 years of age [14]. The high neonatal variability together with the slow progress in acuity development during the neonatal period, seems to complicate assessment of minor differences between various subgroups, in particular if small numbers of infants are being compared.

\section{Acknowledgements}

A.J.M. Hermans was supported by Praeventiefonds No. 28-1430, 1, The Netherlands. We would like to thank M. Blijenberg-Mulder, W. Dell, C. Eveleens Maarse-Havermans, Dr D.J. Heersema, H. de Man-Sonneveld, for their help in testing the infants, B.W.F. Weyer for technical assistance and Dr Th. Stijnen for his advice on the statistical analysis. We greatly acknowledge the friendly cooperation of the nursing staff of the Zuiderziekenhuis.

\section{References}

1 Arduini, D., Rizzo, G., Romanini, C. and Mancuso S. (1987): Fetal blood flow velocity waveforms as predictors of growth retardation. Obstet. Gynecol., 70, 7-10.

2 Atkinson, J. and Braddick, O.J. (1989): Newborn contrast sensitivity measures: do VEP, OKN and FPL reveal differential development of cortical and subcortical streams? Invest. Ophthalmol. Vis. Sci., 30 (Suppl.), 313.

3 Atkinson, J., Braddick, O. and French, J. (1979): Contrast sensitivity of the human neonate measured by the visual evoked potential. Invest. Ophthalmol. Vis. Sci., 18, 210-213.

4 Baraldi, P., Ferrari, F., Fonda, S., and Penne, A (1981): Vision in the neonate: (full-term and premature): preliminary results of the application of some testing methods. Doc. Ophthalmol., 51, $101-112$.

5 Bekedam, D.J., Visser, G.H.A., de Vries, J.J., and Prechtl, H.F.R. (1985): Motor behaviour in the growth retarded fetus. Early Hum. Dev., 12, 155-165.

6 Birch, E.E., Birch, D.G., Petrig, B. and Uauy, R. (1990): Retinal and cortical function of very low birth weight infants at 36 and 57 weeks postconception. Clin. Vision Sci., 5, 4, 363-373.

7 Brown, A.M. and Yamamoto, M. (1986): Visual acuity in newborn and preterm infants, measured with grating acuity cards. Am. J. Ophthalmol., 102, 245-253.

8 Dobson, V., McDonald, M.A. and Teller, D.Y. (1985): Visual acuity of infants and young children: Forced-choice preferential looking procedures. Am. Orthopt. J., 35, 118-125.

9 Dobson, V., Schwartz, T.L., Sandstrom, D.J., Michel, L (1987): Binncular visual acuity of nennates: the acuity card procedure. Dev. Med. Child Neurol., 29, 199-206. 
10 Dobson, V., D'Antonio, J.A. and Bonvalot, K. (1989): Grating acuity in early infancy predicts grating acuity at age 1 year in infants with perinatal complications. Invest. Ophthalmol. Vis Sci., 30 (Suppl.), 142.

11 Dubowitz, L.M.S., Dubowitz, V., Goldberg, C. (1970): Clinical assessment of gestational age in the newborn infant. J. Pediatrics, 77, $1-10$.

12 Fantz, R.L. (1958): Pattern vision in young infants. Psychol. Rec., 8, 3-47.

13 Groenendaal, F., Van Hof-van Duin, J., Fetter, W.P.F., Groot, L de, Hage, M. (1989): Effects of perinatal hypoxia on visual development during the first year of (corrected) age. Early Hum. Dev., 20, 267-279.

14 Heersema, D.J. and Van Hof-van Duin, J. (1990): Age norms for visual acuity in toddlers using the acuity card procedure. Clin. Vis. Sci., 5, 167-174.

15 Kloosterman, G.J. (1970): On intrauterine growth. Int. J. Gynaecol. Obstet., 8, 6, part 2, 895-912.

16 Mc.Donald, M.A., Dobson, VI, Sebris, S.L., Baitch, L, Varner, D. and Teller, D.Y. (1985): The Acuity Card Procedure: A Rapid Test of Infant Acuity. Invest. Ophthalmol. Vis Sci., 26, $1159-1162$.

17 Mohn, G. and Van Hof-van Duin, J. (1986): Rapid assessment of visual acuity in infants and children in a clinical setting, using acuity cards. Doc. Ophthalmol. Proc. Scr., 45, 363-372.

18 Mohn, G., and Van Hof-van Duin, J. (1991): In: Vision and Visual Dysfunction, Vol. 10, Ch. 7, pp. 179-211, Spatial vision. Editor: D.M. Regan, McMillan Press Library, London.

19 Mohn, G., Van Hof-van Duin, J., Fetter, W.P.F., De Groot, L. and Hage, M. (1988): Acuity assessment in non-verbal infants and children: Clinical experience with the acuity card procedure. Dev. Med. Child Neurol., 30, 232-244.

20 Norcia, A.M. and Tyler, Ch.W. (1985): Spatial frequency sweep VEP: visual acuity during the first year of life. Vision Res., 25 (10), 1399-1408.

21 Norcia, A.M., Tyler, Ch.W., Piecuch, R., Clyman, R. and Grobstein, J. (1987): Visual acuity development in normal and abnormal preterm human infants. J. Pedr. Ophthal. Strab., 24, 2, 70-74.

22 Paneth, N., Kiely, J.L., Wallenstein, S., Marcus, M., Pakter, J. and Susser, M. (1982): Newborn intensive care and neonatal mortality in low-birth weight infants. N. Engl. J. Med., 307 (3), 149-155.

23 Porciatti, V., Vizzoni, L., van Berger, G.P. (1982): Neurological age determination by evoked potentials. In: Paediatric Ophthalmology, pp. 345-348. Editor: F.J. Maione M. John Wiley and Sons and Cortine Ve.

24 Van den Wijngaard, J.A.G.W., Groenenberg, I.A.L., Wladimiroff, J.W. and Hop, W.C.J. (1989):

Cerebral Doppler ultrasound of the human fetus. Br. J. Obstet. Gynaecol., 96, 845-849.

25 Van Hof-Van Duin, J. and Mohn, G. (1986): The development of visual acuity in normal fullterm and preterm infants. Vision Res., 26 (6), 909-916.

26 Van Hof-van Duin, J., Mohn, G., Fetter, W.P.F., Mettau, J.W. and Baerts, W. (1983): Preferential looking acuity in preterm infants. Behav. Brain Res., 10, 47-51.

27 Van Hof-van Duin, J., Mohn, G., Groenendaal, F. and Evenhuis-van Leunen, A (1987): Visual acuity of newborn infants assessed with gratings and checkerboard patterns. Behav. Brain Res. 26, 242-243.

28 Van Hof-van Duin, J., Evenhuis-van Leunen, A., Mohn, G., Baerts, W. and Fetter, W.P.F. (1989): Effects of very low birth weight (VLBW) on visual development during the first year after term. Early Hum. Dev., 20, 255-266.

29 WHO: recommended definitions, terminology and format for statistical tables related to the perinatal period and use of a new certificate for cause of perinatal deaths. (1977). Acta Obstet. Gynecol. Scand., 56, 247-253. 